\title{
A detailed comparison of antenna impedance measurements at ASDEX Upgrade with the ICRF antenna code TOPICA
}

\author{
I Stepanov ${ }^{1}, \mathbf{J}$ M Noterdaeme ${ }^{1,2}, \mathrm{~V}$ Bobkov $^{2}, \mathbf{H}_{\text {Faugel }^{2}}, \mathbf{D}$

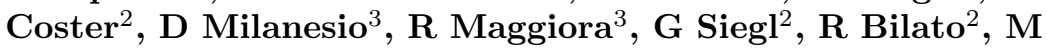 \\ Brambilla $^{2}$, G Verdoolaege ${ }^{1,4}, \mathbf{F}$ Braun $^{2}$, H F ünfgelder $^{2}, \mathbf{R}$ \\ D'Inca $^{2}$, W Suttrop ${ }^{2}$, A Kallenbach ${ }^{2}$, J Schweinzer ${ }^{2}$, E \\ Wolfrum $^{2}$, R Fischer ${ }^{2}$, A Mlynek ${ }^{2}$, V Nikolaeva ${ }^{2,5,6}$, L \\ Guimarais $^{6}$ and the ASDEX Upgrade team ${ }^{2}$ \\ ${ }^{1}$ Department of Applied Physics, Ghent University, Sint-Pietersnieuwstraat 41, \\ B-9000 Ghent, Belgium \\ ${ }^{2}$ Max Planck Institute for Plasma Physics, EURATOM Association, \\ Boltzmannstrasse 2, D-85748 Garching, Germany \\ 3 Department of Electronics and Telecommunications, Politecnico di Torino, \\ Corso Duca degli Abruzzi 24, 10129 Torino, Italy \\ ${ }^{4}$ Laboratoire de Physique des Plasmas de l'ERM - Laboratorium voor \\ Plasmafysica van de KMS (LPP-ERM/KMS), Ecole Royale Militaire - \\ Koninklijke Militaire School, Avenue de la Renaissance 30, B-1000 Brussels, \\ Belgium \\ 5 Technische Universität München, James-Franck-Str. 1, D-85748 Garching, \\ Garching \\ ${ }^{6}$ Instituto de Plasmas e Fusão Nuclear, Instituto Superior Técnico, \\ Universidade de Lisboa, Av. Rovisco Pais, 1049-001 Lisboa, Portugal \\ E-mail: ivan.stepanov@ugent.be
}

\begin{abstract}
New antenna diagnostics on ASDEX Upgrade, in the form of voltage and current probe pairs on the feeding lines of each ICRF antenna, close to the input ports, has made it possible to study in detail the behavior of the ASDEX Upgrade two-strap antenna under changing loading conditions, and compare these measurements with results of simulations using the TOPICA code. The present work extends previous studies by using the input impedance (more precisely, the complex voltage reflection coefficient $\Gamma$ ) on each antenna port for comparison, instead of the more commonly used loading resistance or coupled power. The electron density profiles used for the simulation were reconstructed from DCN interferometer and Lithium beam emission spectroscopy measurements, ELMsynchronized and averaged over time intervals from 10 to $200 \mathrm{~ms}$ depending on the case; 112 cases were compared from seven ASDEX Upgrade discharges with widely different plasma parameters and two operating frequencies (30 and 36.5 $\mathrm{MHz}$ ). Very good agreement in $|\Gamma|$ is found with the measurements on antenna 3 ( $<3 \%$ averaged over a shot), good agreement is found with antennas 1 and $2(<10 \%)$; the code reproduces the correct trend in loading resistance $R_{L}$ in a significant majority of cases, although the discrepancies in the absolute values can be rather high (up to $\sim 50 \%$ ) due to high reflection. Sources of discrepancy are discussed.
\end{abstract}

PACS numbers: 52.50.Qt 
Keywords: ICRF, coupling, antenna impedance

Submitted to: Nucl. Fusion 


\section{Introduction}

Determining the reliability of antenna modeling tools is crucial for the task of predicting the power coupling capability of the ITER ICRF (ion cyclotron range of frequencies) antenna. One of the state-of-the art codes used to evaluate the ITER antenna performance is TOPICA (TOrino POlytechnic Ion Cyclotron Antenna), which handles an arbitrarily shaped three-dimensional antenna model [1], facing a hot, multispecies plasma in slab geometry described by a modified version of the FELICE (Finite ELement Ion Cyclotron Emulator) code, which includes finite Larmor radius (FLR) effects up to second order in the ratio of the ion Larmor radius to the typical perpendicular wavelength [2]. In recent years, TOPICA has been extensively tested against experimental results obtained on Alcator C-Mod [1], Tore Supra [3], DIII-D [4], JET [5] and ASDEX Upgrade [6]; it is not straightforward to directly compare the results of these studies, since they differ in the quantities being compared: coupling resistance per unit length in Tore Supra, effective conductance in JET, complex voltage reflection coefficient in Alcator C-Mod, loading resistance in DIII-D; in the case of ASDEX Upgrade, a voltage reflection coefficient measured behind the matching network was used (in addition two other studies investigated the performance of both the baseline and the wide limiter antenna at ASDEX Upgrade in terms of coupling and RF potentials, namely [7] and [8], but no comparisons with experiment are quoted). The agreement with experimental data has usually been rather good. The Alcator C-Mod study reported $<10 \%$ agreement in $|\Gamma|$ and $<10^{\circ}$ in the phase of $\Gamma$ (in many cases better, see [1] for details); the JET and Tore Supra studies both report TOPICA results well within the measurement error bars (in the latter the coupling to the plasma has been evaluated by integrating the RF current over the strap length, see [3] for details). The DIII-D study reports very good absolute agreement, especially in H-mode [4]. In the ASDEX Upgrade study, two different diagnostic sets were used: voltage probe arrays on unmatched feeding lines and directional couplers behind the matching network (see Section 2); the results ranged from moderately good $(\sim 10 \%$ in $|\Gamma|)$ with directional couplers to inadequate $(\sim 20 \%$ in $|\Gamma|)$ with voltage probe arrays, with both sets of diagnostics showing a large (up to $\sim 90^{\circ}$ ) phase discrepancy (details can be found in [6]).

The aim of this paper is extend and improve the last TOPICA validation study on ASDEX Upgrade by making use of newly implemented RF diagnostics, which reduce some of the uncertainties associated with the use of directional couplers located behind the matching network, while also giving access to some previously unavailable information about antenna behavior. In addition, a significantly wider variety (and higher number) of density conditions are investigated here.

\section{The ASDEX Upgrade ICRF system and diagnostics}

The ICRF system at ASDEX Upgrade consists of four two-strap antennas (antennas 1 - 3 being baseline [9] and antenna 4 being a wide limiter, narrow strap antenna designed to reduce RF sheaths and hence $\mathrm{W}$ sputtering [10]). The four antennas were connected into two double systems, where the output power of two RF generators was combined and then split into two $50 \Omega$ lines by means of $3 \mathrm{~dB}$ couplers for ELM protection (details can be found in [11] and [12]); each line was connected to a matching section (consisting of two parallel tuning stubs) and split into two $25 \Omega$ lines feeding the two antenna straps. The antennas are typically operated in $(0, \pi)$ phasing at 30 and 36.5 


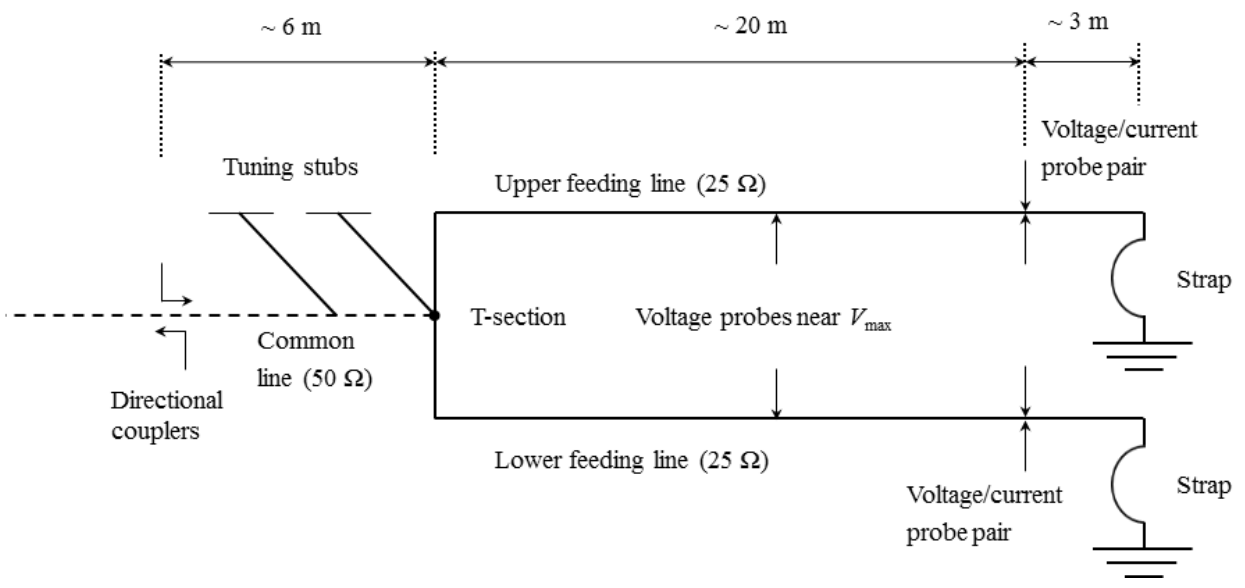

Figure 1. Antenna diagnostics on one (of four) ASDEX Upgrade transmission network. Lengths are approximate and different for each system. $V_{\max }$ refers to the voltage maximum on each line. Not shown are the voltage probe arrays on each $25 \Omega$ feeding line, which are not used in this study. Note that this schematic is only meant to show the available diagnostics and main features of each system, and does not show the real strap geometry (which is examined in detail in [9]).

$\mathrm{MHz}$, corresponding to the fundamental ion cyclotron frequencies of hydrogen when the toroidal magnetic field on axis is 2 and $2.5 \mathrm{~T}$ respectively. The details of the setup can be found in [13]; the network section including the common line, matching network, individual feeding lines and straps, and the relevant RF diagnostics is shown schematically in figure 1 . Note that the straps are not fed at the same poloidal location, and the current facing the plasma flows in opposite directions when the ports are fed with the same phasing (see [9] for details).

The directional couplers and $V_{\max }$ probesł, which are located near the estimated position of the voltage maximum on each line, constitute the standard diagnostics, from which the loading resistance is normally computed via the definition [14]

$$
R_{L}=\frac{2 Z_{0}^{2} P_{c p l}}{V_{\max }^{2}}
$$

where $P_{c p l}$ is the coupled power, $Z_{0}$ the characteristic impedance of the line (in this case $25 \Omega$ ) and $V_{\max }$ is the voltage measured by the voltage maximum probe. The newly implemented diagnostics, in the form of voltage/current probe pairs installed close to the antenna ports on the feeding lines, provide information on input impedance $Z_{\text {inp }}$, voltage reflection coefficient $\Gamma$, loading resistance $R_{L}$ and coupled power $P_{c p l}$ on each individual line by measuring the RMS voltage and current magnitudes ( $V$ and $I$ resp.) at the same point on the line and the phase $\theta$ between them, giving

$$
Z_{i n p}=\frac{V}{I} \mathrm{e}^{j \theta} \quad \Gamma=\frac{Z_{i n p}-Z_{0}}{Z_{\text {inp }}+Z_{0}} \quad R_{L}=\frac{Z_{0}}{V S W R} \quad P_{c p l}=V I \cos \theta
$$

with $V S W R=(1+|\Gamma|) /(1-|\Gamma|)$. One of the main reasons for using this setup is that measurements are made close to the antenna input; thus, in contrast with the previous study, accurate knowledge of the transmission network up to the T-section and behind

$\ddagger$ The voltage probe array consists of about 10 probes on each line, only one of which is the voltage maximum probe; the rest of the array was not used in the present study. 
is not needed. Some preliminary results with the voltage and current probes on ASDEX Upgrade have been reported: antenna 1, upper line (uncalibrated) [15], and antenna 3, both lines [6]. Since 2011 all probes have been calibrated, integrated into the data acquisition system and used during both routine operation and dedicated RF experiments on ASDEX Upgrade.

All signals are read by logarithmic $\mathrm{RF}$ detectors with a dynamic range of $60 \mathrm{~dB}$ and a time resolution of $5 \mu \mathrm{s}$.

Calculation of $Z_{i n p}$ or $\Gamma$ using voltage and current probes is extremely sensitive to errors in $\theta$ introduced by differences in the length of the cables connecting the two probes to the RF detector, which in this case is $\sim 100 \mathrm{~m} \S$. Although this difference was measured with a network analyzer (with a nominal accuracy of $2^{\circ}$ ), subsequent analysis of routinely measured reflection coefficients revealed that correction factors obtained in this way were a few degrees off the expected values. In order to find the missing corrections, the coupled power calculated from probe measurements was compared to the power evaluated from directional coupler measurements in a series of shots in which the antennas were assumed to operate in a well-matched regime and in which, therefore, the errors in transmitted power measurements due to the finite directivity of the couplers were deemed to be low. Because the two power measurements (RF probes and directional couplers) are done at locations far away from each other, resistive losses in the transmission network were taken into account, although with line resistivity in the range of $10^{-3}-10^{-2} \Omega / \mathrm{m}$ (at $36.5 \mathrm{MHz}$ ) the losses along both feeding lines amount to $1 \%-2 \%$ of coupled power. The additional assumption of equal loading resistance on the two straps (ELM-synchronized and averaged over each shot) was also used (see Appendix A for details). In other words, for each shot corrections were added to the phases $\theta_{u p}$ and $\theta_{l w}$ so that the loading on both straps were equal $\left(R_{L, u p}=R_{L, l w}\right)$ and the sum of the coupled power measured on both lines by the probes is equal to the coupled power measured on the common line by the directional couplers, i. e. $P_{l w}+P_{u p}=P_{D C}$ minus resistive losses.

The corrections obtained in this way constitute a distribution with mean values in the range $0.8^{\circ}-8.9^{\circ}$, depending on antenna and operating frequency and with standard deviations in the range $0.1^{\circ}-3.6^{\circ}$, again depending on antenna and frequency. The correction finally implemented for each pair of probes, i. e., for each feeding line, was the average over the whole series of well-matched shots and it was this value that was used consistently for all the shots analyzed below\|. On the other hand, the standard deviations, together with the known accuracy of $0.6 \mathrm{~dB}$ in probe coupling values, were used to estimate the corresponding standard deviation $\sigma$ for $|\Gamma|, \Phi(\Gamma)$ and $R_{L}$. The error bars shown for the results below correspond to $\pm 3 \sigma \boldsymbol{q}$.

We note that the determination of coupled power from directional coupler measurements does not require knowing the antenna input impedance at the T-section, and therefore it is not sensitive to precise knowledge of the tuning stub settings, circumventing that particular source of error.

$\S \mathrm{RF}$ detectors are extremely sensitive to external noise and are located in the shielded ICRF control room.

\| The finite width of this distribution of values means that, as a rule, the upper and lower reflection coefficients $\Gamma_{u p}$ and $\Gamma_{l w}$ will be close but not identical, as is indeed seen in the results presented in section 4 .

II It can certainly be argued that instead of phase, coupling corrections for $V$ or $I$ could be introduced; however this leads to very large corrections $(\sim 2 \mathrm{~dB})$ which are deemed to be less plausible than phase errors corresponding to a few centimeters of cable length. 

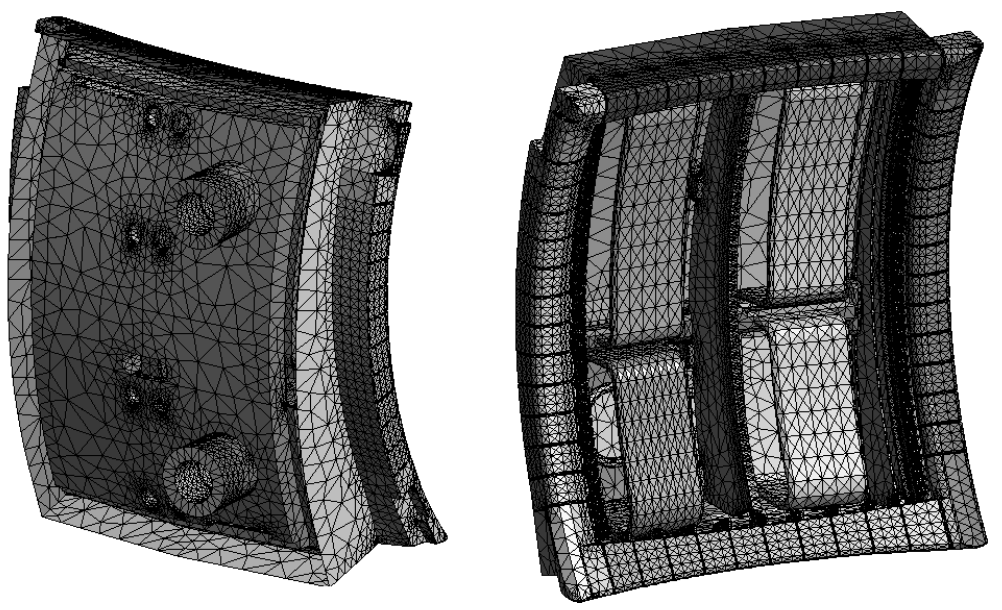

Figure 2. Model of the ASDEX Upgrade antenna used in the simulation; Faraday screen bars omitted for clarity. Upper and lower input ports seen on the back. Triangular mesh with 68,628 triangles is shown.

\section{Simulation setup and input data}

A fully curved model of the ASDEX Upgrade baseline two-strap antenna was used for the simulation; the model and the corresponding triangular mesh with 68,628 triangles are shown in figure 2. It is an accurate representation of antennas 1 and 2, having an open Faraday screen; antenna 3 differs from the model somewhat in that it has perforated straps and, at the time the measurements were taken, it had an optically thick Faraday screen (changed in 2014). Antenna 4 is electrically and mechanically different, as mentioned in section 2 , and will be included in a future study. In all simulations the antenna feeding scheme corresponded to the normally used $(0, \pi)$ strap phasing.

Besides from the antenna model, input data consisted of electron density $n_{e}$ and temperature $T_{e}$ profiles. Electron density is measured in the core and pedestal top via DCN interferometer and at the edge/SOL by Lithium beam emission spectroscopy; electron temperature is measured by electron cyclotron emission. Point-wise profiles along the whole plasma radius are reconstructed via Bayesian analysis [16] and are available with a time resolution of $1 \mathrm{~ms}$; the resolution of the density profile in the edge region is $5 \mathrm{~mm}$ (densities up to $0.8 \times 10^{20} \mathrm{~m}^{-3}$ are accessible by Lithium beam [16]). For each shot, time windows ranging from 10 to $200 \mathrm{~ms}$ were selected. Because of the large fluctuations in density due to ELMs, input profiles were ELM-synchronized where needed (i. e., in all H-mode shots except 29990; see table 1). The outer divertor current was taken as indicator of ELM activity and only the $n_{e}$ and $T_{e}$ profiles between 3.5 and $1.5 \mathrm{~ms}$ before each ELM event were considered; all the profiles available in these intervals within each window were averaged into one density and one temperature profile and used as input. Likewise, only experimental data within the same intervals was averaged and used for comparison (see section 6 for details and illustrations). The $n_{e}$ and $T_{e}$ profiles are cut at $R_{m a j}=2.2 \mathrm{~m}$, which is the radial position of the antenna limiters at the midplane. Toroidal invariance of the $n_{e}$ and $T_{e}$ profiles was assumed for this study (see section 5 for a discussion of this assumption) and therefore only one FELICE run (and one TOPICA run) was used for comparison with all three antennas 


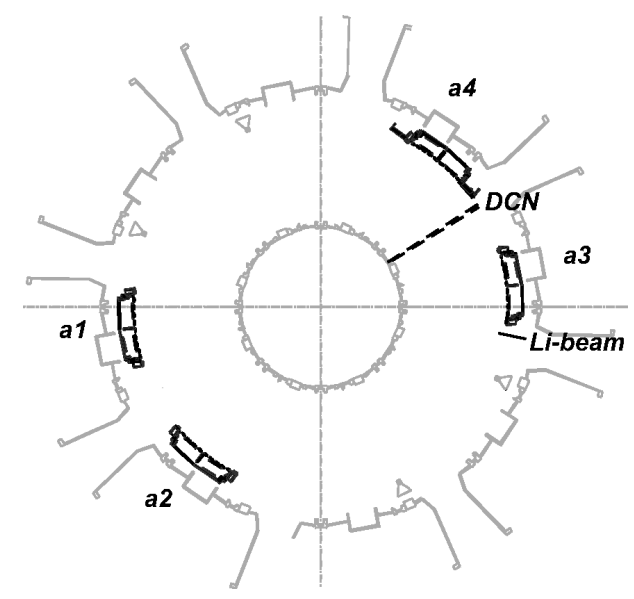

Figure 3. Toroidal location of the ICRF antennas 1-4, DCN interferometer and diagnostic Lithium beam on ASDEX Upgrade.

Table 1. ASDEX Upgrade discharges used in this study; $\delta_{o}$ is upper triangularity. In 29990, ELMs were suppressed with non-axisymmetric magnetic perturbations.

\begin{tabular}{lllll}
\hline Shot $\mathrm{nr}$. & $B_{0}(\mathrm{~T})$ & $f(\mathrm{MHz})$ & $\mathrm{RF}$ data & Remarks \\
\hline 29093 & 2 & 30.0 & $\mathrm{~A} 3$ & L-mode with neutral gas injection \\
29110 & 2.5 & 36.5 & $\mathrm{~A} 3$ & L-mode with $15 \% \mathrm{H}, 85 \% \mathrm{D}$ \\
29113 & 2.5 & 36.5 & $\mathrm{~A} 3$ & L-mode with $47 \% \mathrm{H}, 53 \% \mathrm{D}$ \\
29822 & 2.5 & 36.5 & $\mathrm{~A} 1, \mathrm{~A} 2, \mathrm{~A} 3$ & H-mode with strong $\delta_{o}$ variation \\
29823 & 2.5 & 36.5 & $\mathrm{~A} 1, \mathrm{~A} 2, \mathrm{~A} 3$ & H-mode with strong $\delta_{o}$ variation \\
29833 & 2 & 30.0 & $\mathrm{~A} 1, \mathrm{~A} 2, \mathrm{~A} 3$ & H-mode with strong $\delta_{o}$ variation \\
29990 & 2.5 & 36.5 & $\mathrm{~A} 2, \mathrm{~A} 3$ & H-mode with ELM suppression \\
\hline
\end{tabular}

at each time point.

The numbers and main characteristics of the discharges during which profiles and $\mathrm{RF}$ measurements were recorded are summarized in table 1. Unless stated otherwise, plasma composition was taken to be $1.5 \% \mathrm{H}, 98.5 \% \mathrm{D}$. Although this is somewhat lower than the usual $\mathrm{H}$ concentration in ASDEX Upgrade $(\sim 5 \%)$, sensitivity tests showed that varying the $\mathrm{H}$ content from $0 \%$ to $10 \%$ had virtually no effect on the results. Note that for shot 29990, no A1 RF data is available.

\section{Results}

In order to speed up the computation, only a small portion of the transmission network is included in the TOPICA model (it was verified that a distance of $\sim 15 \mathrm{~cm}$ from any discontinuity is enough to assume that the electric field inside the coaxial line recalls the ideal TEM feeding distribution adopted in TOPICA); the $2 \times 2$ scattering matrix $\mathbf{S}$, which constitutes the relevant part of the TOPICA output, is used instead to compute the quantities of interest by the familiar equations of transmission line theory ${ }^{+}$. We note that the matrix $\mathbf{S}$ is normalized to a (real) reference impedance

+ For the purposes of comparing TOPICA with experimental data, the ultimate goal would be to measure the full antenna scattering matrix. Such measurements are described in [17], [18] and [19]. 
of $25 \Omega$, which is the characteristic impedance of the feeding lines. To obtain the reflection coefficients on both ports, the scattering matrix should be first translated towards the antenna input (i. e. the feeder-strap interface), to eliminate the effect of the $\sim 15 \mathrm{~cm}$ of coaxial line added in TOPICA:

$$
\mathbf{S}^{\prime}=\mathbf{\Theta S} \Theta
$$

where

$$
\boldsymbol{\Theta}=\left[\begin{array}{cc}
e^{j \beta l_{1}} & 0 \\
0 & e^{j \beta l_{2}}
\end{array}\right],
$$

$\beta=2 \pi f / c$, and $l_{1}, l_{2}$ are the distances to the input ports along the lower and upper lines respectively $(+14.8 \mathrm{~cm})$. By the definition of the scattering matrix,

$$
\vec{V}^{-}=\mathbf{S}^{\prime} \vec{V}^{+}
$$

where $\vec{V}^{+}=\left[V_{1}^{+} V_{2}^{+}\right]^{\mathrm{T}}$ is the vector containing the incident voltage waves and $\vec{V}^{-}=\left[V_{1}^{-} V_{2}^{-}\right]^{\mathrm{T}}$ is the vector containing the scattered waves for ports 1 and 2 respectively. Feeding the array with unitary voltage waves $\vec{V}^{+}=\left[\begin{array}{ll}1 & 1\end{array}\right]^{\mathrm{T}}$ produces the reflection coefficients

$$
\Gamma_{i}=\frac{V_{i}^{-}}{V_{i}^{+}}
$$

We recall here again that, as mentioned in section 2 , in order to have a current distribution with a $(0, \pi)$ phasing the waves incident on the ports have the same phase. Note that these are active reflection coefficients, because both ports are fed simultaneously. Here we remark that, in the case of strong cross-coupling, the real feeder voltages would have an effect on the measurements of reflection coefficient on the neighboring line. In the TOPICA S-matrices however the off-diagonal terms are much smaller than the diagonal terms; if one introduces significant deviations from the dipole phasing into the feeding scheme, up to $20^{\circ}$ in phase and $\sim 20 \%$ amplitude, the results are still within the error bars of the RF measurements. We note also the TOPICA assumes that all antenna surfaces are perfect conductors and the code does not take resistive losses into account.

Finally, since the voltage/current probes are located some $3 \mathrm{~m}$ from strap input, reflection coefficients measured at their location were translated towards the antenna input (feeder-strap interface) in order to carry out the comparison at the same reference plane as the TOPICA results.

The results of the comparison with data from shot 29093 are shown in figure 4 (only data for antenna 3 are available for shots 29093, 29110 and 29113 as no probes had been implemented on the other systems). Only the reflection coefficient is shown for clarity here for each shot; the loading resistance $R_{L}$ of each strap computed with TOPICA, for all the studied cases, is plotted versus the corresponding measured values in figure 20 at the end of this section. The increase and subsequent decrease in $|\Gamma|$ correspond to a scan in separatrix position, which is shown in figure 5 . The decrease in $|\Gamma|$ and increase in the phase of $\Gamma$ (denoted by $\Phi=\Phi(\Gamma)$ ), corresponding to a decrease of the electrical length of the strap, is similar to what is observed during an ELM burst in [20].

The agreement in antenna coupling - here given by $|\Gamma|$ - is very good: the average of the relative difference

$$
\frac{\Delta|\Gamma|}{|\Gamma|}=100 \times\left|\frac{|\Gamma|_{\text {TOPICA }}-|\Gamma|_{\text {meas }}}{|\Gamma|_{\text {meas }}}\right|
$$



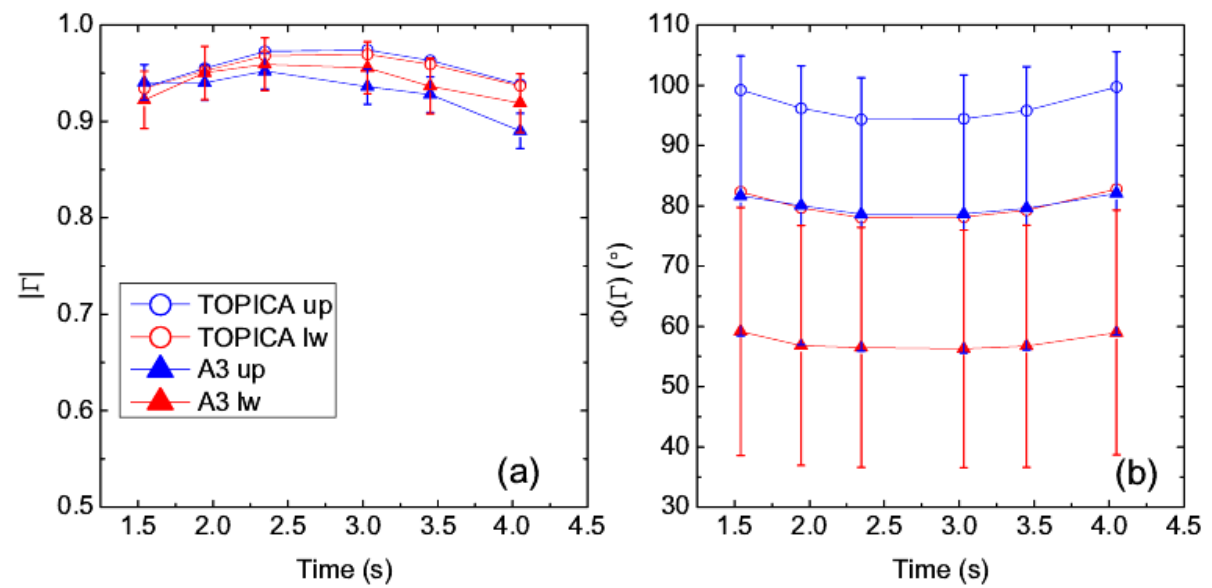

Figure 4. Magnitude (a) and phase (b) of $\Gamma$, shot 29093, antenna 3.

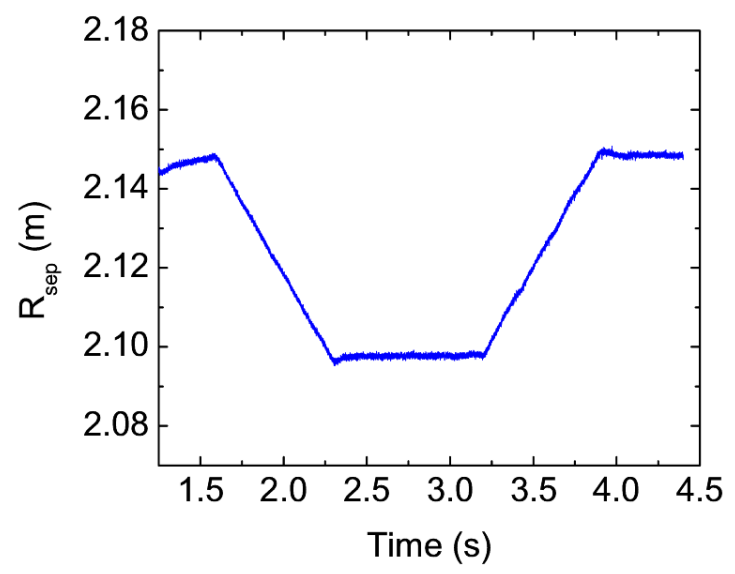

Figure 5. Separatrix position time trace, shot nr. 29093.

is $2.9 \%$ for the upper line and $1.4 \%$ for the lower line.

It can also be seen that there is a relatively large (but roughly constant) difference in the phase of the reflection coefficient between the simulated and measured results, i. e. $\Delta \Phi=\Phi($ TOPICA $)-\Phi$ (measured); the average values are $17^{\circ}$ and $23^{\circ}$ (upper and lower lines respectively). If caused by an error in line length, at $30 \mathrm{MHz}$ they correspond to equivalent lengths of 23 and $31 \mathrm{~cm}$. Since the length of the lines between the probe location and the antenna port is around $3 \mathrm{~m}$, this amounts to an error on the order of $10 \%$ of that length*. Furthermore, we note that the line section between the probe pair location and the antenna input port is highly non-uniform due to the presence of the vacuum feedthrough and the transition to the strap, making it difficult to accurately measure the line length between the probes and the antenna input. This is therefore a plausible cause of the observed discrepancy in $\Phi(\Gamma)$. We note that the effect of these sections can be extracted by manipulating their scattering matrices,

* Note that the phase $\Phi$ of the reflection $\Gamma$ is different from the phase $\theta$ of the input impedance $Z_{i n p}$, defined in equation 2 . 

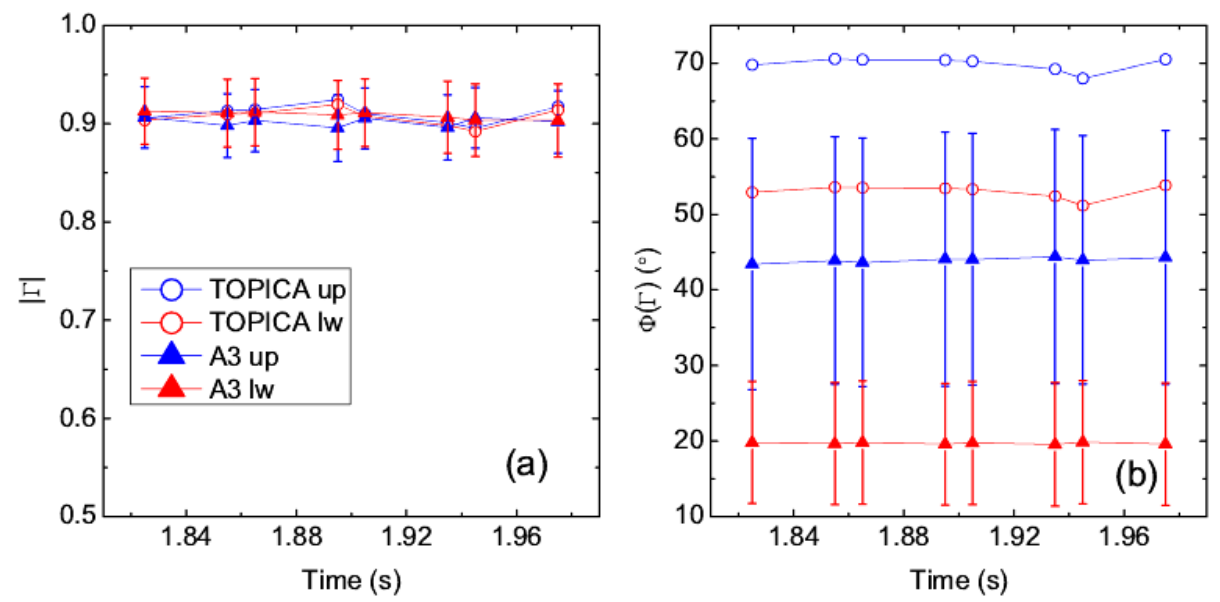

Figure 6. Magnitude (a) and phase (b) of $\Gamma$, shot 29110, antenna 3.

which could reduce the associated length errors; while the matrices were not available for the present work, full-wave modeling of these line sections is to be included in future work.

Figures 6 and 7 show the results from shots 29110 and 29113. In contrast to the other shots presented here, no significant variation in coupling is seen because no changes were introduced in the plasma (no separatrix shift, for instance); the data was included in the study because of the non-standard plasma composition (see table 1). Relative errors in coupling are in the same range as in 29093. The average $\Delta \Phi$ values are $26^{\circ}, 33^{\circ}$ (s. 29110 , corresponding to 30 and $38 \mathrm{~cm}$ at $36.5 \mathrm{MHz}$ ) and $26^{\circ}, 32^{\circ}$ (29113, corresp. to 30 and $37 \mathrm{~cm}$ ), which is reasonably close to the differences seen in 29093, thereby increasing the likelihood that they are indeed due to a length error. We note also that the decrease in the phase of $\Gamma$, when going from 30 to $36.5 \mathrm{MHz}$, can be anticipated from transmission line calculations, since translating a reflection coefficient $\Gamma$ by a distance $d$ away from the load amounts to a clockwise rotation by $2 \beta d$ radians.

Shots 29822, 29823 and 29833 were part of a series to test antenna behavior with changing upper plasma triangularity $\delta_{o}$; therefore the variation in density - hence in antenna loading - is much more significant than in the previous shots. Figure 8 shows the time traces of the separatrix position $R_{s e p}$ and upper triangularity $\delta_{o}$. Three $R_{s e p}$ scans were performed (roughly between 2-3, 4-5 and 6-7 s). Lower triangularity (not shown) $\delta_{u}$ stays at $\sim 0.45$ while $\delta_{o}$ changes from $\sim 0.1$ to $\sim 0.32$.

In addition to antenna 3, data from antennas 1 and 2 had become available. Figures 9,10 and 11 show the time variation of the measurements and simulation results during shot 29822 for antennas 1, 2 and 3 respectively. Noticeable in the measurement time traces (and somewhat less in the simulation results) are two of the three $R_{\text {sep }}$ scans (roughly between $2-3$ and $4-5 \mathrm{~s}$ ) and a significant decrease in $|\Gamma|$ around $\sim 5.5 \mathrm{~s}$, where $\delta_{o} \simeq 0.29$. From figures 9,10 and 11 it is evident that the data from antennas 1 and 2 agree less with the code results than antenna 3 . This is notable since the 3D geometry of the TOPICA model corresponds to the one of antennas 1 

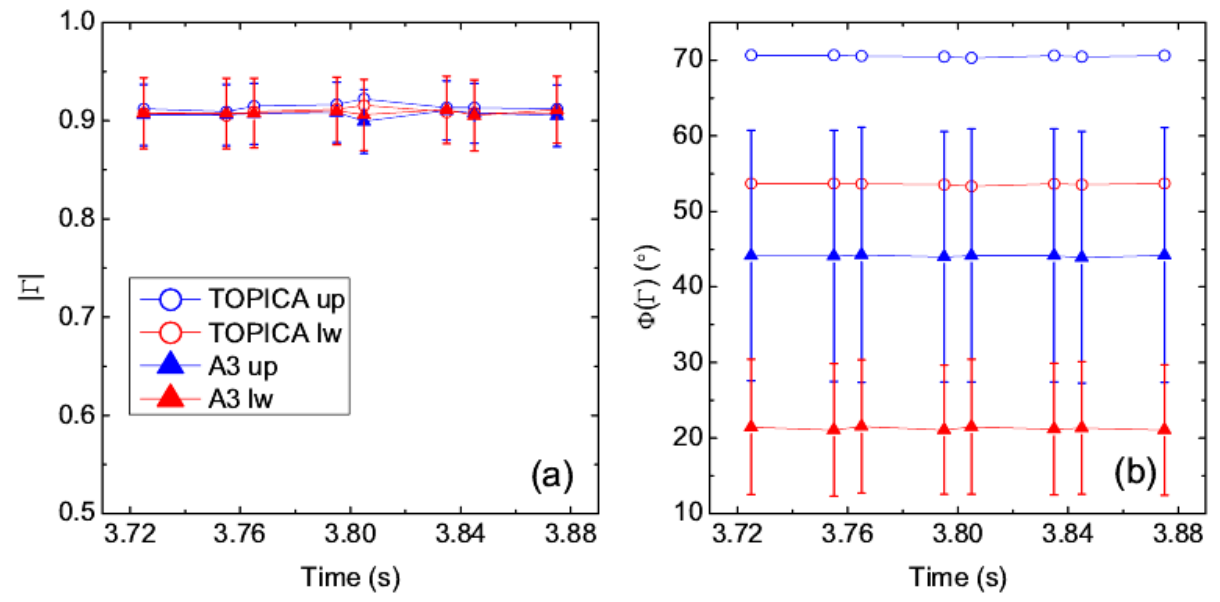

Figure 7. Magnitude (a) and phase (b) of $\Gamma$, shot 29113, antenna 3.

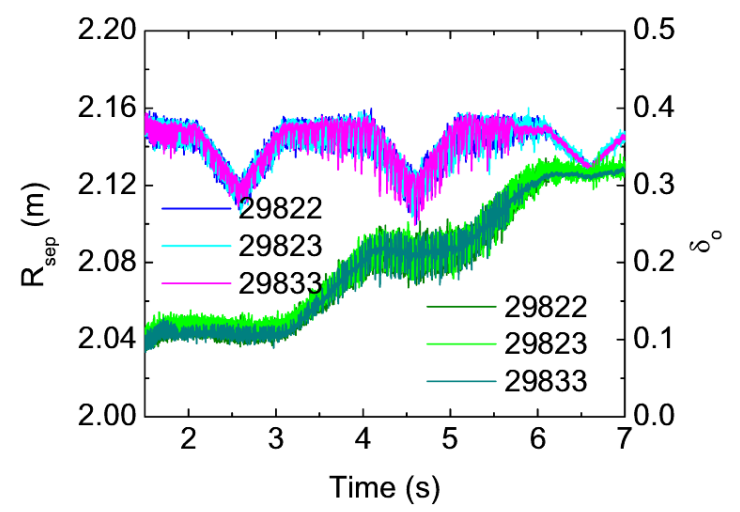

Figure 8. Time traces of separatrix position $R_{s e p}$ and upper triangularity in shots 29822,29823 and 29833.

and 2 (which have open Faraday screens $\sharp$ ). The reasons for this observation are not yet known and tentative explanations are discussed in section $5 \dagger \dagger$.

For antenna 1, the average relative errors in $|\Gamma|$ are $9.3 \%$ and $6.5 \%$ (upper and lower line resp.), and for antenna $29.0 \%$ and $6.8 \%$ (in contrast, on antenna 3 the corresponding numbers are $2.2 \%$ and $1.8 \%$ ). Nonetheless the code follows fairly well the general trend in loading throughout the discharge, with the exception of the anomalous downward peak roughly between 5.1 and $5.7 \mathrm{~s}$ noticeable on both magnitude and phase plots, with no similar peak being observed on the experimental data. The other major discrepancy, as stated, is observed from $\sim 6 \mathrm{~s}$ to the end of the discharge (see discussion below). Notably, the phase differences also increase somewhat towards the end of the discharge, although not as drastically as the loading;

\# Although antenna coupling should be better with an open FS, this is not enough to explain the large difference in loading between antennas 1, 2 and 3.

$\dagger \dagger$ From the phase of $\Gamma$ on figures 9,10 and 11, it can be seen that antenna 3 is also electrically shorter than antennas 1 and 2, but it is not clear if the reason is related to the Faraday screen (a simulation with the optically thick Faraday screen will provide a clearer answer). 

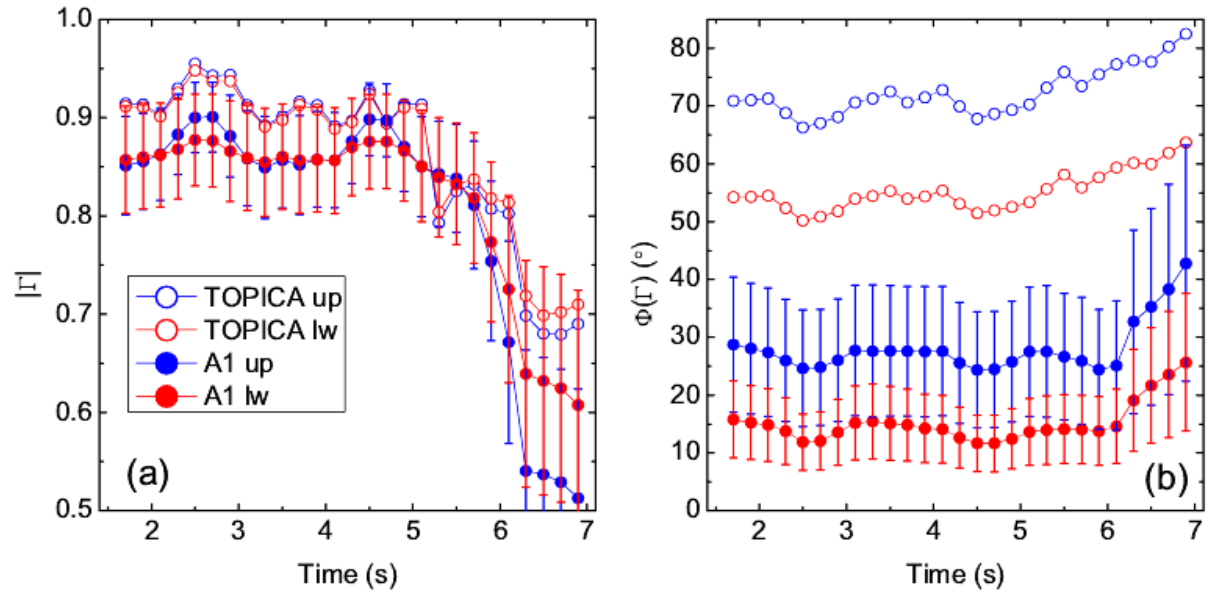

Figure 9. Magnitude (a) and phase (b) of $\Gamma$, shot 29822, antenna 1.
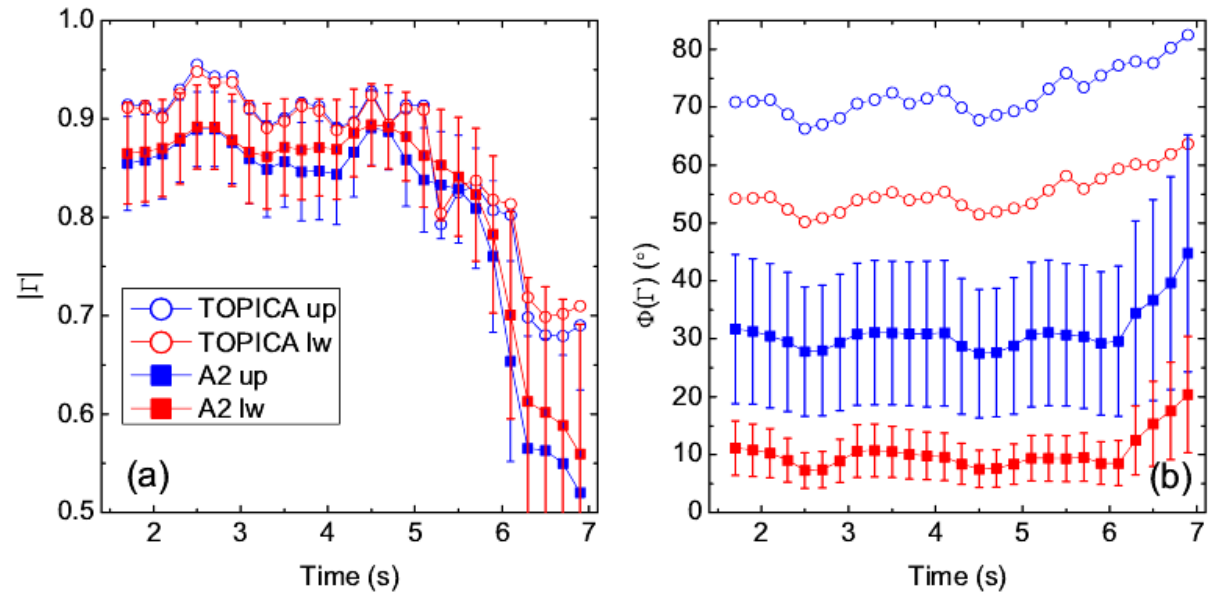

Figure 10. Magnitude (a) and phase (b) of $\Gamma$, shot 29822, antenna 2.

this is consistent with the observations from shot 29093.

The comparison results from shot 29823 are shown in figures 12 and 13 for antennas 1 and 3 respectively (the results for antenna 2, as for shot 29822, are very similar to antenna 1 and are omitted here for brevity). The conclusion again is that the code shows remarkable agreement with antenna 3 , even after $6 \mathrm{~s}$ at high $\delta_{o}$ : the average $\Delta|\Gamma| /|\Gamma|$ is $1.8 \%$ and $1.6 \%$, on the upper and lower lines. For antennas 1 and 2 the corresponding numbers are $5.7 \%, 5.4 \%$ and $5.5 \%, 3.3 \%$ respectively - which is significantly better than in shot 29822 - and the code reproduces well the main trend in loading. The situation is less clear where the phase of $\Gamma$ is concerned. In both shots, the phase of each reflection coefficient calculated with TOPICA increases visibly after $\sim 5 \mathrm{~s}$ (this is much clearer in shot 29822); on the other hand, the phase of the measured $\Gamma$ increases in 29822 but actually decreases somewhat in 29823 , leading to an overall increase in $\Delta \Phi$ throughout the discharge. 

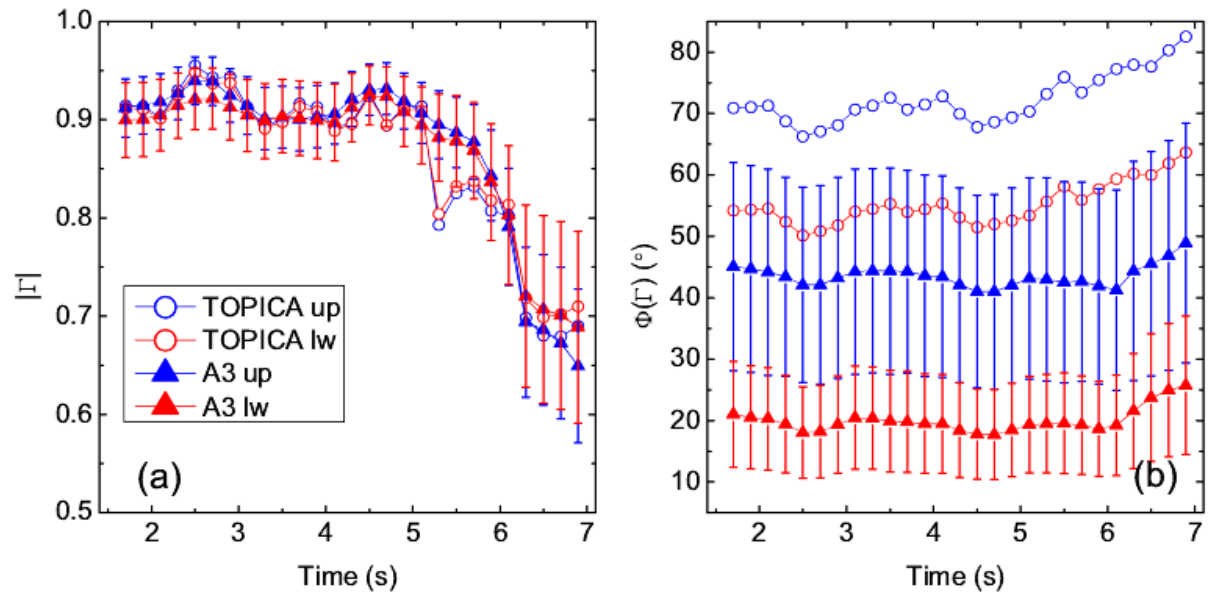

Figure 11. Magnitude (a) and phase (b) of $\Gamma$, shot 29822, antenna 3.
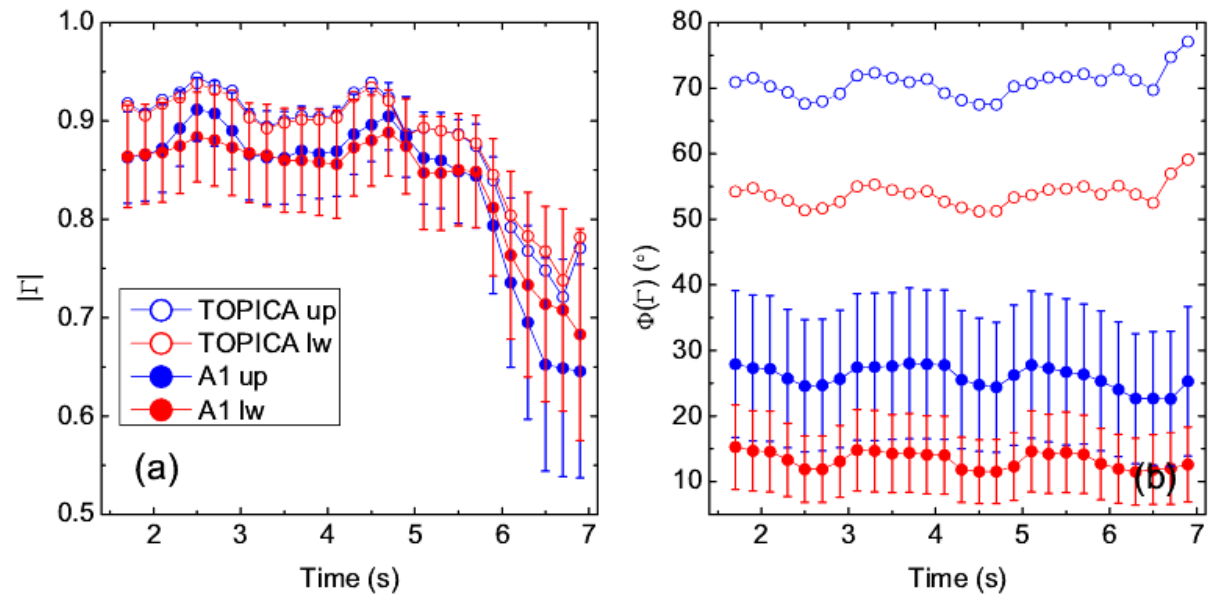

Figure 12. Magnitude (a) and phase (b) of $\Gamma$, shot 29823, antenna 1.

A possible explanation for the divergence between TOPICA and experimental measurements towards the end of both discharges could be that the high $\delta_{o}$ conditions observed in this interval lie outside the validity domain of the slab plasma geometry used in TOPICA; however, figure 8 shows that $\delta_{o}$ was the same for all shots, and as shown, the discrepancy is much smaller in the results of shot 29823. Furthermore, changes in plasma shape are illustrated for shot 29822 in figure 14, along with the poloidal positions of the diagnostics used for density profile reconstruction, i. e. the DCN interferometer and the Lithium beam. From figure 14, it can be seen that there is no substantial change in plasma shape at midplane from 6 to 7 seconds, despite the large variations in coupling observed in the same time interval shown in figures 9, 10 and 11 (the observations are similar for shot 29823 and not illustrated here for brevity). We therefore conclude that the discrepancies between measurements and TOPICA results seen at high $\delta_{o}$ in these shots are at least not due to the use of the FELICE slab approximation to simulate highly shaped plasmas. 

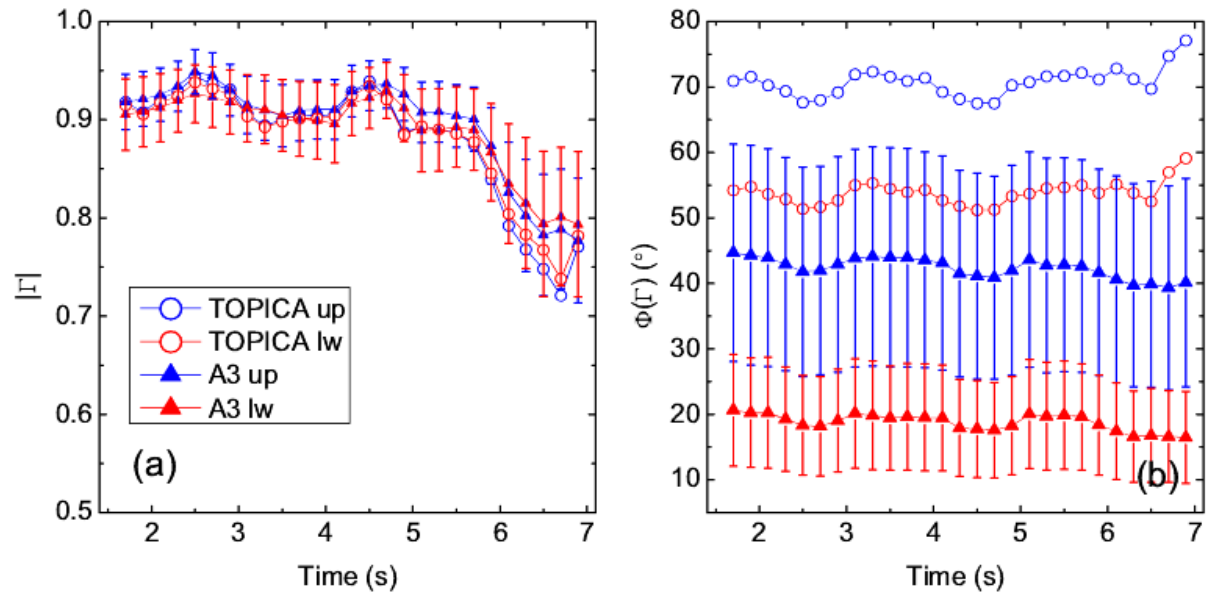

Figure 13. Magnitude (a) and phase (b) of $\Gamma$, shot 29823, antenna 3.

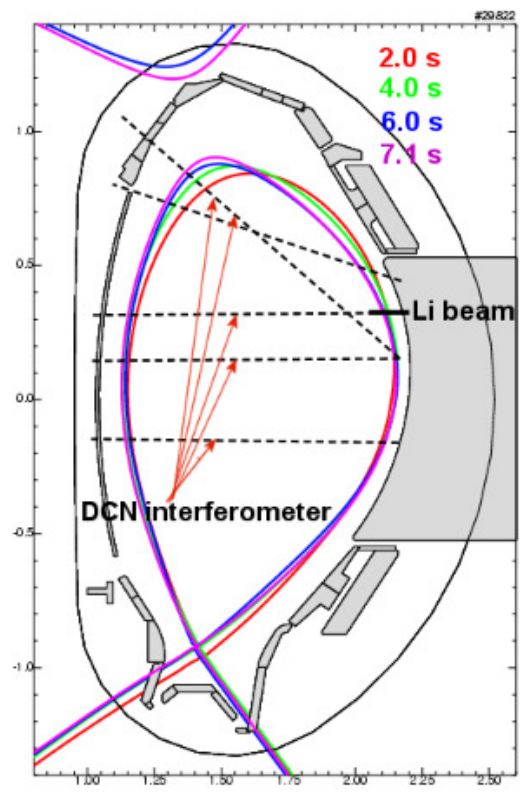

Figure 14. Variations in separatrix shape at several times during shot 29822. The poloidal location of the diagnostics used for the reconstruction of the density profile is also shown.

On the other hand, a global density rise is associated with the $\delta_{o}$ increase; to illustrate this, eight representative density profiles used as FELICE input are shown in figure 15, four from shot 29822 and four from shot 29823 (the profiles were obtained as described in section 3). The radial position of the antenna limiter and the fast wave cutoff ( $\sim 3 \times 10^{18} \mathrm{~m}^{-3}$ in the ASDEX Upgrade case) are also shown. In the last two cases of each shot, where $|\Gamma|$ is significantly decreased, the edge density is well above cutoff. TOPICA assumes that no plasma is present in the antenna box, which therefore results in an abrupt change in $n_{e}$ from zero to some relatively high value, which is 


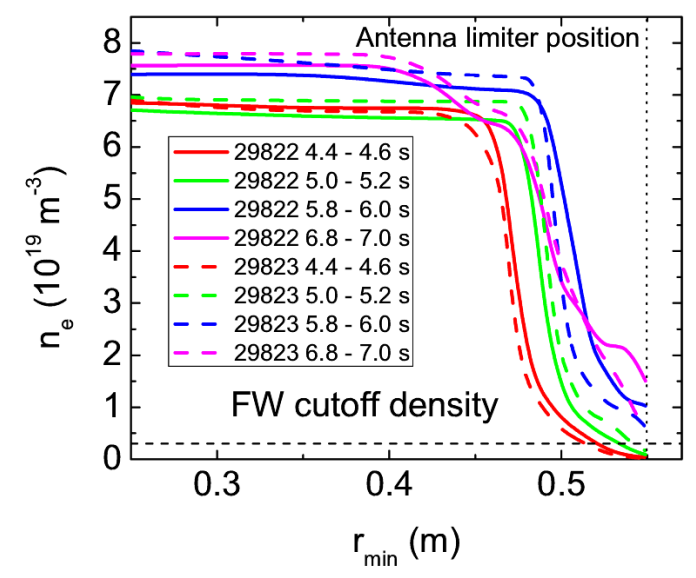

Figure 15. IDA reconstruction of four representative $n_{e}$ profiles from shots 29822 and 29823, used in FELICE. All profiles are ELM-synchronized and averaged over the indicated time window. The radial position of the antenna limiter and the approximate fast wave cutoff density $\left(\sim 3 \times 10^{18} \mathrm{~m}^{-3}\right)$ are also shown. Note that in the last two cases in both shots, the edge $n_{e}$ is significantly above cutoff density. This causes an abrupt change in density at the aperture of the antenna cavity, since TOPICA assumes there is no plasma in the cavity; this in turn leads to a possible overestimation of $|\Gamma|$ at high $\delta_{o}$.

expected to degrade coupling (this is shown with an analytical model in [21] and [22]). However, in reality the density is nonzero inside the antenna cavity, decreasing the gradient and also allowing the fast wave to propagate; therefore under these conditions the measured $|\Gamma|$ is expected to be lower than calculated. In other words, TOPICA models a lower coupling because it does not take into account the density inside the antenna box, while in the illustrated shots the cutoff is located in that region. This is consistent with the observation that the edge $n_{e}$ at 5.8 and 6.8 seconds is significantly lower in 29823 than in 29822, with a corresponding better agreement between TOPICA and experiment (the same argument applies to shot 29833). Because of the restrictions of the TOPICA model, extending the $n_{e}$ profile closer to the antenna would require removing the limiter, thereby reducing the geometrical accuracy of the comparison. Nonetheless this hypothesis is to be tested in further work.

In shot 29833 the same procedure was followed as in 29822-29823 $\left(\delta_{o}\right.$ increase and $R_{\text {sep }}$ scans, as shown in figure 8) but with a central field of $2 \mathrm{~T}$ and with an operating frequency of $30 \mathrm{MHz}$. The data from all antennas follow the same general trend as before. The results of the comparison are shown in figures 16 and 17 (antenna 2 results are very similar to antenna 1 and are again omitted for brevity). $\Delta|\Gamma| /|\Gamma|$ is in the same range as in previous shots. The agreement is again very good for antenna 3 ; however a difference in the trend of $|\Gamma|$ is seen roughly from 3.3 to $4 \mathrm{~s}$, with a small peak occurring in the TOPICA results, again with no similar peak occurring on any antenna; this is more clearly seen on antenna 1 (figure 16) and is consistent with a brief shift of the IDA density profile away from the antenna.

We remark at this point that the significant coupling improvement towards the end of shots 29822, 29823 and 29833 is strongly correlated with the neutral flux density measured by an ionization gauge located in the main chamber midplane, and hence a rise in SOL density. The reason for this is not yet clear, although it 

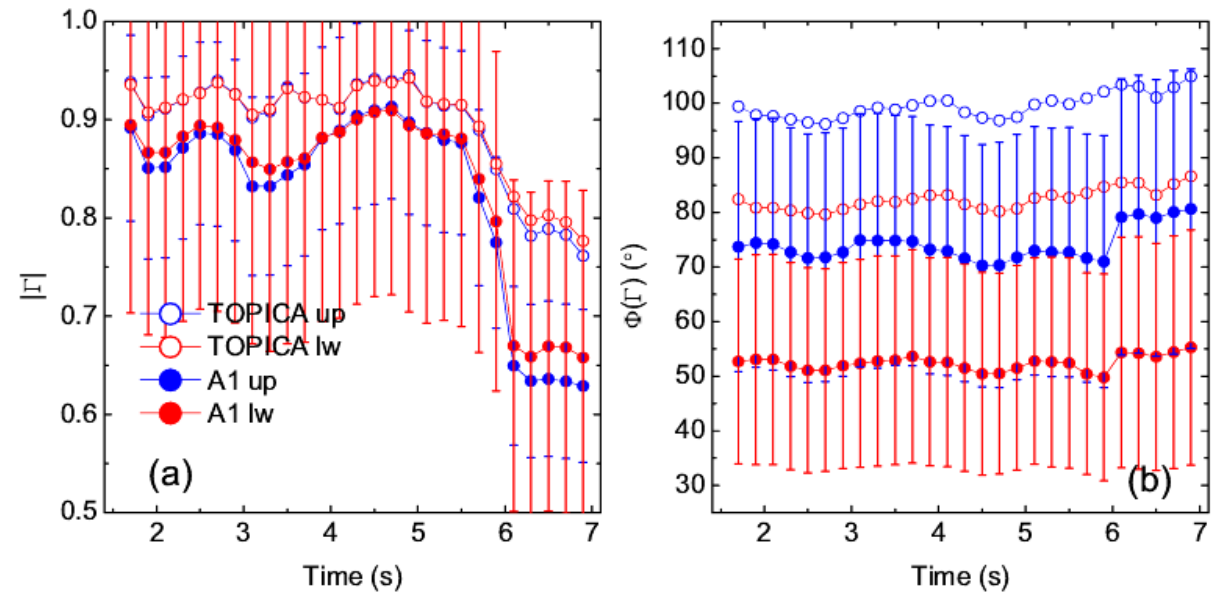

Figure 16. Magnitude (a) and phase (b) of $\Gamma$, shot 29833, antenna 1.
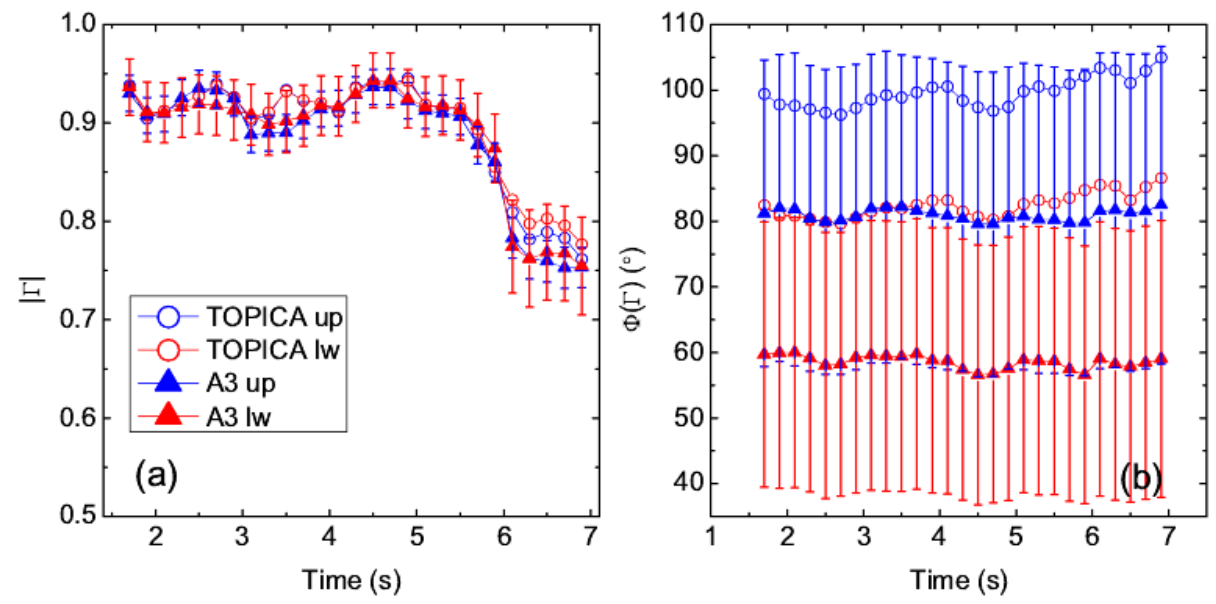

Figure 17. Magnitude (a) and phase (b) of $\Gamma$, shot 29833, antenna 3.

is known that triangularity variations can affect gas recycling through changes in the connection length of the magnetic field lines in the SOL [13]. There is however no direct link between triangularity and coupling, unless the plasma becomes poloidally inhomogeneous in front of the straps; the main factor affecting the coupling is the density profile in front of the antenna, consistent with the conclusions in [23] and [22].

Finally, the results for shot 29990 are shown in figures 18 and 19. Once again the code reproduces very well the overall trend in loading, although the variation is not as high as in other shots. This particular pulse with suppressed ELMs was included in the study to look for a possible impact of the absence of ELMs on the TOPICA results (while retaining H-mode density profile in contrast to 29093, 29110 and 29113); furthermore, density profile modification has also been observed when using non-axisymmetric perturbation fields for ELM mitigation (such as in the discharge included here) on ASDEX Upgrade [24]. However, no clear effect is seen in the results presented here. 
Although the complex voltage reflection coefficient $\Gamma$ is well suited for a detailed antenna study, in the sense that comparing a complex quantity can reveal more information than, e. g., loading resistance $R_{L}$ which is a function of $|\Gamma|, R_{L}$ is much more frequently used as a figure of merit for antenna coupling properties. The loading resistance for each strap is obtained from the TOPICA results by

$$
R_{L, i}=Z_{0} \frac{1-\left|\Gamma_{i}\right|}{1+\left|\Gamma_{i}\right|},
$$

in accordance with equation 2 and using the active reflection coefficients defined by equation 6 . The calculated loading for all shots, as function of measured loading, is shown in figure 20. It is evident that the agreement with antenna 3 is very good, while a substantially higher (than modeled by TOPICA) loading is observed on antennas 1 and 2. Note that approximately the same $R_{L}$ is measured on both, which would be expected because they are located next to each other and therefore must face the same plasma.

It must also be noted that, because of the usually very high VSWR on the feeding lines during normal operation, even moderate differences in $|\Gamma|$ mean rather large relative differences in $R_{L}$. This is seen from equation 9 , using $\rho=\left|\Gamma_{i}\right|$ :

$$
\frac{\partial R_{L}}{\partial \rho}=-\frac{2 Z_{0}}{(1+\rho)^{2}} \Rightarrow \frac{\Delta R_{L}}{R_{L}}=-\frac{2 \Delta \rho}{1-\rho^{2}}
$$

for small $\Delta \rho$ and $\Delta R_{L}$. For example, in shot 29823, for antenna 1 the average $\Delta|\Gamma| /|\Gamma|$ values are found to be $5.7 \%$ and $5.4 \%$ on the upper and lower lines, respectively, which translate into relative errors in $R_{L}$ of $30 \%$ and $31 \%$ respectively. Therefore we stress that examining reflection coefficients strongly damps the discrepancies between the code and the experimental results. The alternative is to compare the real and imaginary parts of the complex impedance $Z_{i n p}$; however, this has the disadvantage that errors in line length will affect both quantities and make the analysis less clear (the relationship between $\Gamma$ and $Z_{i n p}$ is given in equation 2). Furthermore, we choose to examine $\Gamma$ in the present work to be consistent with the previous ASDEX Upgrade study [6]. Nonetheless, we underline that the caveat regarding the large errors in $R_{L}$ must be kept in mind, since this is ultimately the quantity that coupled power is proportional to (see equation 1 ).

\section{Discussion and conclusions}

The differences between measurements and simulation results, for all shots, are summarized in figure 21. Each point is the average over the whole corresponding shot. $\Delta \Phi$ is given in centimeters as equivalent mechanical length. As stated at the end of the last section, with respect to coupling (i. e. $|\Gamma|$ or $R_{L}$ ) the simulation results show excellent agreement with the experimental data from antenna 3 , and good agreement with antennas 1 and 2 . While the trend is correct, antennas 1 and 2 exhibit a substantially higher loading than computed by the code, almost by a factor of 2 at high $R_{L}$. For all antennas, the disagreement becomes larger at high density, due to the restrictions of the TOPICA model as discussed in section 4. Regarding phase, it can be seen that $\Delta \Phi$ varies only by a few centimeters from shot to shot, therefore making it likely that this discrepancy is caused by a systematic error in line length measurement.

The reason for the large differences in loading for the three antennas is still unknown; several hypotheses can be invoked as possible explanations, but a clear 

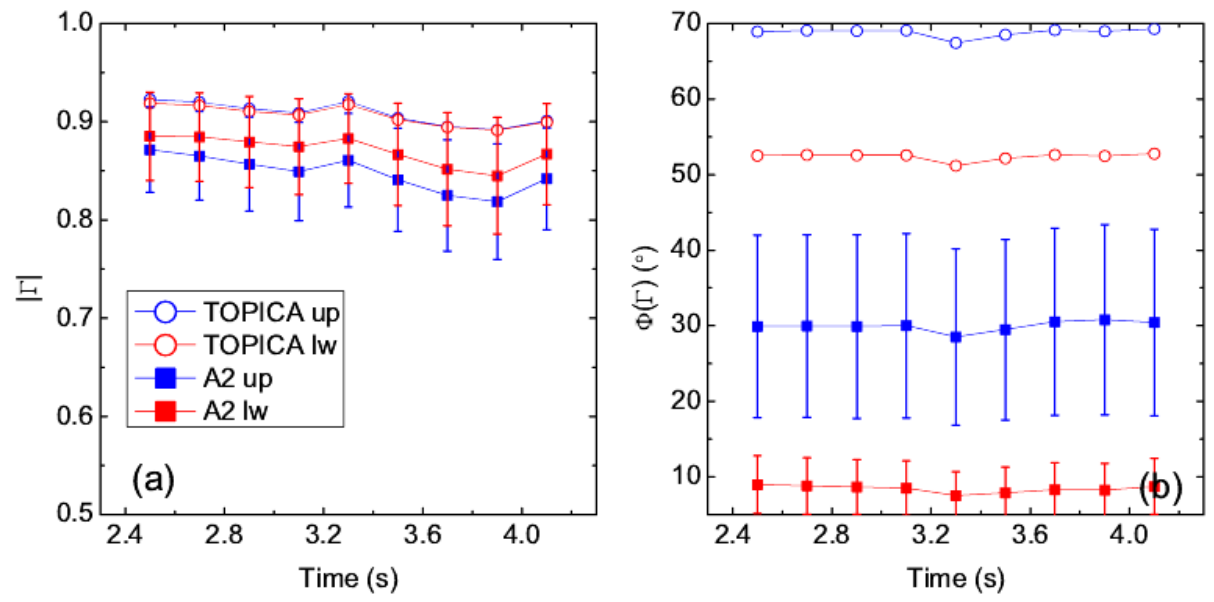

Figure 18. Magnitude (a) and phase (b) of $\Gamma$, shot 29990, antenna 2.
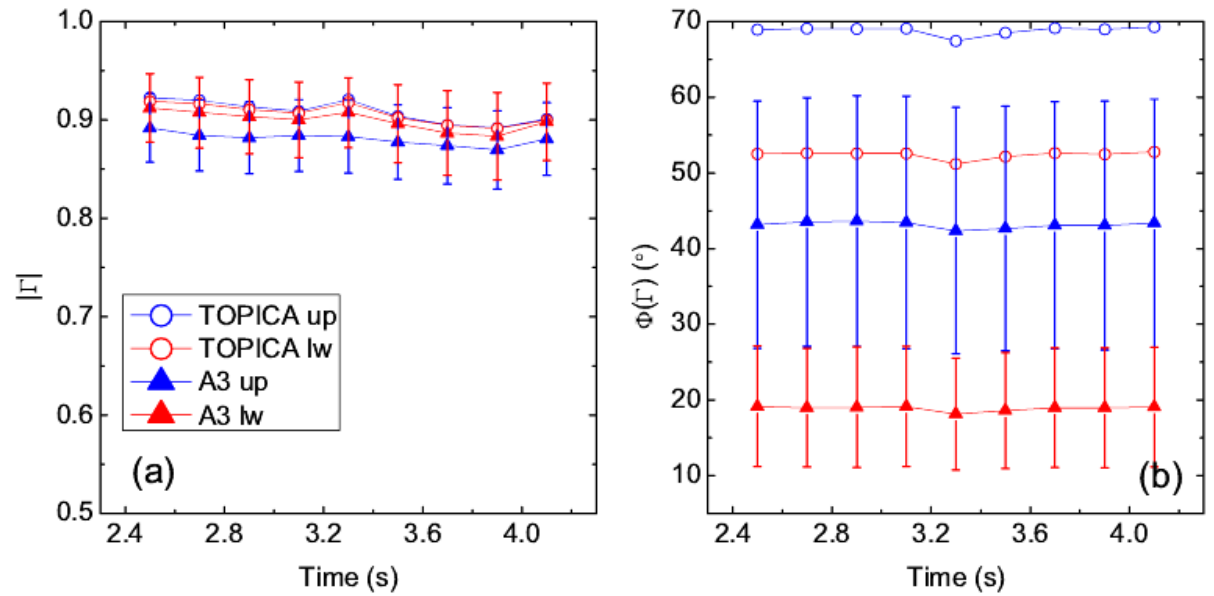

Figure 19. Magnitude (a) and phase (b) of $\Gamma$, shot 29990, antenna 3.
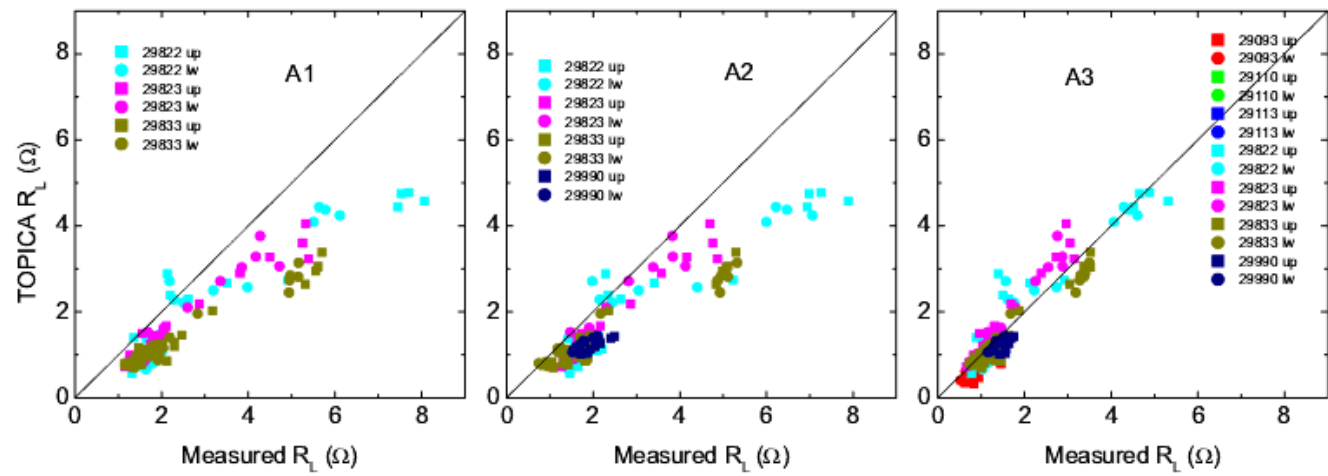

Figure 20. Results for all shots, antennas 1, 2 and 3. 


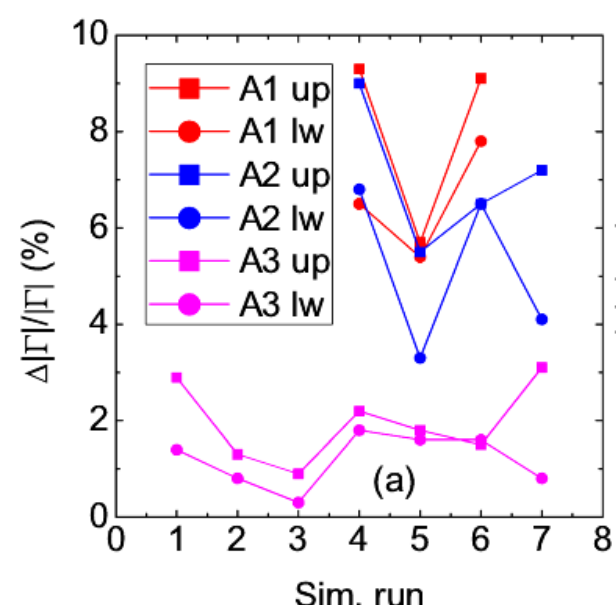

Sim. run

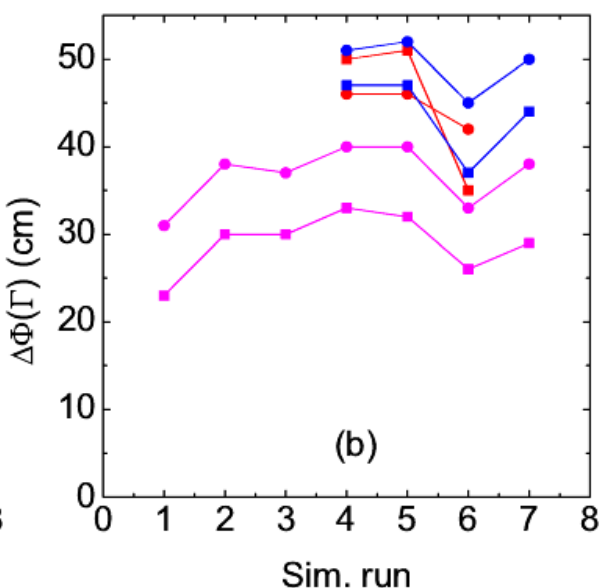

Figure 21. Overall differences between measurement and simulation results. $\Delta \Phi$ is given in centimeters as equivalent mechanical length; the horizontal axis indicates the shot number from 29093 (1) to 29990 (7).

reason has not yet been found. First, the diagnostic Lithium beam that measures the edge $n_{e}$ profile is located in sector 9 , in close proximity to antenna 3 (sector 10) and far from antennas 1 and 2 (sectors 2 and 4 resp., essentially on the opposite side of the torus); on the other hand there is no strong reason to believe that the plasma had any significant toroidal asymmetry. Magnetic perturbation coils were switched on from 1.15 until 4.10 seconds in shot 29990, encompassing almost the whole time window during which plasma profiles and RF data were measured; however, this would not explain the differences between the antennas seen in 29822, 29823 and 29833, as MP coils were on only from 1.7 to 2 seconds in shots 29822 and 29823, and from 1.7 to $3.6 \mathrm{~s}$ in 29833. In any case, a factor of $\sim 2$ is too high to be explained by MPs alone, since their effect on antenna behavior has also been studied on ASDEX Upgrade [25], [26] and less significant effects were reported.

Second, as stated in section 2, at this point correction factors in the phase difference between voltage and current signals still have to be introduced to match the total coupled power to that measured by directional couplers; therefore the measurements depend directly on another diagnostic system. With this in view, it can be argued that it might not be correct to impose the condition of equal loading resistance on the two radiating straps to obtain the correction factors. We note that, strictly speaking, this would only be expected if the the radiating straps were identical (however note that TOPICA predicts a very similar $R_{L}$ for both straps). The effects of relaxing this constraint can be seen, for example, by requiring that the power coupled on both feeding lines be equal instead. Technically this would be expected if the loading resistances were equal and the feeding lines were also equal in length, but given the geometrical complexity of the transmission network the latter is unlikely to be exactly fulfilled; nonetheless it is a useful alternative to look at. With the correction factors thus obtained, one may recompute the measurement result, e. g., for antennas 1 and 2 in shot 29822. The result is shown in figure 22 (only $|\Gamma|$ is plotted). Comparing figures 22 (a) and (b), one can see that there is a substantial difference in coupling between the two antennas, which would be unexpected since, as stated above, antennas 1 and 

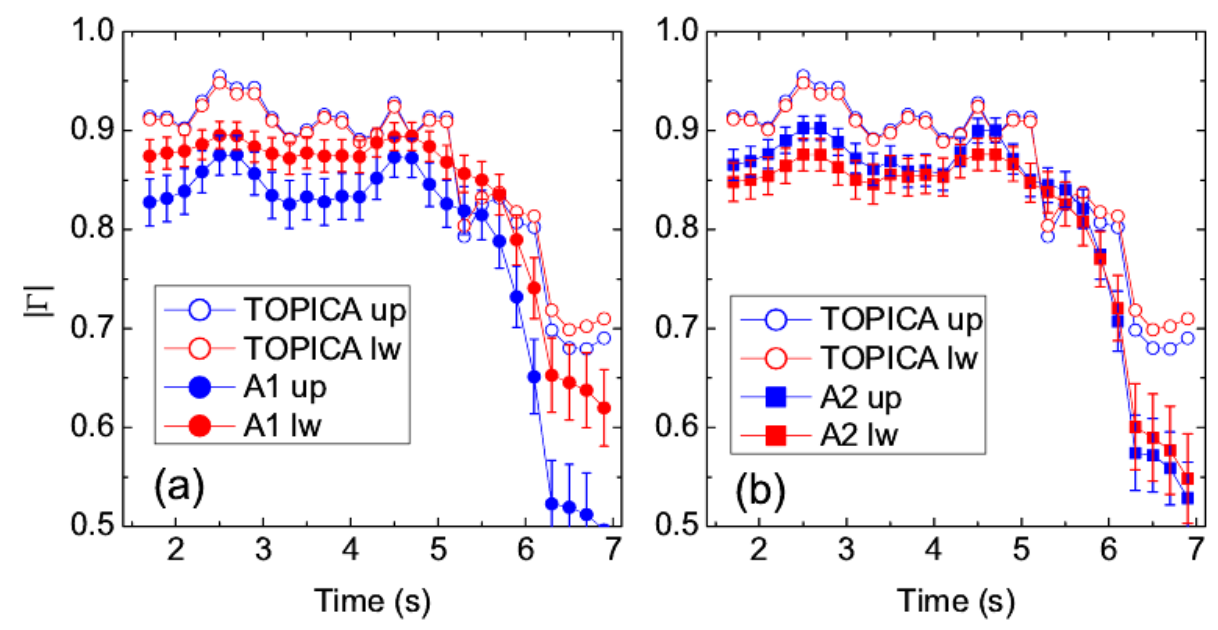

Figure 22. Magnitude of $\Gamma$, antennas 1 (a) and 2 (b), shot 29822, using correction factors calculated with the constraint of equal coupled power on both lines. There are substantial differences in coupling between the two antennas, as well as between the two straps of antenna 1 .

2 are located next to each other and should be facing the same plasma. There is also a significant difference between the two straps on antenna 1, indicating that the assumption of equal loading is probably more realistic; compare 22 (a) with figure 9 , which shows the results with the condition of equal loading and where the two straps of antenna 1 exhibit much less difference. In any case, averaging the $|\Gamma|$ on both lines gives roughly the results shown in figures 9 and 10; therefore it is unlikely that the assumption of equal loading is the reason for the observed discrepancy.

A third reason for the higher loading observed on antennas 1 and 2 could be a systematic error in the radial position of the antenna; as mentioned in section 3 , the same radial position was used in TOPICA for all antennas. As an estimate of this error, we can use the expression

$$
R_{L} \propto P_{0} \exp \left(-1.1 k_{\|} L_{c}\right)
$$

proposed in [21], with $k_{\|}$being the peak of the antenna current spectrum (in the case of ASDEX Upgrade $\simeq 7.7 \mathrm{~m}^{-1}$ ) and $L_{c}$ the distance from the plasma surface to the R-cutoff. An increase of $R_{L}$ by a factor of 2 - roughly the difference in loading between antennas 1 and 3 seen from figure 20 - would correspond to a radial shift of about $8 \mathrm{~cm}$, a rather large difference. (Naturally such a large uncertainty in measurement would also cast doubt on the results of antenna 3.)

For the reasons outlined above, none of these hypotheses offers a clear explanation for the discrepancies, and further analysis is needed. As the absolute comparison of TOPICA results with measured impedances depends crucially on the reliability of the RF diagnostics, subsequent work will be focused on finding the reason for the phase correction factors described in section 2 , with the aim of making the voltage/current probes fully independent from the standard diagnostics. Nonetheless, the use of the probes in this study - as well as making measurements much closer to the antenna, without the additional complexities of the transmission network - shows a significant improvement over the results of the TOPICA comparison in [6] (where directional couplers and voltage probe arrays were used) and therefore places smaller error bars 
on the code results, which are given by $\Delta|\Gamma| /|\Gamma|$ and $\Delta \Phi(\Gamma)$ in figure 21. Despite the shortcomings, it is notable that the code shows such a close agreement with the antenna positioned closest to the locations of the diagnostic Lithium beam and DCN interferometer; these results stress once again the need to measure the density profile as close to the antenna as possible, for any ICRF antenna code study.

\section{Acknowledgements}

This work has been carried out within the framework of the EUROfusion Consortium and has received funding from the European Union's Horizon 2020 research and innovation programme under grant agreement number 633053. The views and opinions expressed herein do not necessarily reflect those of the European Commission.

\section{Appendix A}

Coupled power is measured in the standard diagnostic set by directional couplers located behind the matching network, (see figure 1 for details), so it is separated from the point at which coupled power is measured by the probes by about $30 \mathrm{~m}$ of line length, on which some fraction is lost due to finite resistivity. In the low-loss approximation [27], the propagation constant $j \beta$ is to be replaced by $\gamma=\alpha+j \beta$, where $\alpha \simeq R Z_{0} / 2$ accounts for losses and $R$ is the resistance per unit length of the line. The coupled power measured at the T-section is related to the coupled power measured on the two feeding lines, at the location of the probes, by [27]

$$
P_{T}=\sum_{i=O, U} \frac{P_{i}\left(1-\left|\Gamma_{i} \mathrm{e}^{-2 \gamma L_{i}}\right|^{2}\right) \mathrm{e}^{2 \alpha L_{i}}}{1-\left|\Gamma_{i}\right|^{2}},
$$

where $O, U$ denote upper and lower lines respectively, $P_{i}$ is the coupled power measured at the probe location, $\Gamma_{i}$ are the reflection coefficients (also at probe location), $L_{i}$ is the line length from the probe location to the T-section. For instance, $R=6.2 \times 10^{-3} \Omega / \mathrm{m}$ at $36.5 \mathrm{MHz}$ (corresponding to $1.1 \times 10^{-3} \mathrm{~dB} / \mathrm{m}$ ); with this value the power lost along a single $25 \Omega$ feeding line is in the range $1 \%-2 \%$ of the power coupled on that line, which is at least an order of magnitude smaller than the typical error bars on coupled power calculated from probe measurements. For the $50 \Omega$ stub tuners and the transmission line between them $R$ is roughly the same $8.4 \times 10^{-3} \Omega / \mathrm{m}$ at $36.5 \mathrm{MHz}\left(1.5 \times 10^{-3} \mathrm{~dB} / \mathrm{m}\right)$, while the line length is significantly shorter: $\sim 3$ $\mathrm{m}$ between the two matching stubs, $2.1-2.6 \mathrm{~m}$ from the generator side stub to the directional couplers. The average stub lengths for e. g. antenna 1 were $1840 \mathrm{~mm}$ and $3503 \mathrm{~mm}$ (antenna side and generator side resp.) with a maximum variation of $1 \mathrm{~mm}$ from shot to shot, for the H-mode discharges considered here in order to calculate the probe phase corrections (variation of resistive losses was therefore negligible; at $30 \mathrm{MHz}$ and for antennas 1 and 2 the observations are similar and are omitted here for brevity). We also note that the average current on the $25 \Omega$ lines are higher than on the $50 \Omega$ lines and tuners, making the losses on the unmatched lines even more dominant.

Because of these reasons, the power lost on the short stretch between the Tjunction and the directional couplers is not taken into account here; therefore we equate the power measured by the latter to $P_{T}$. 


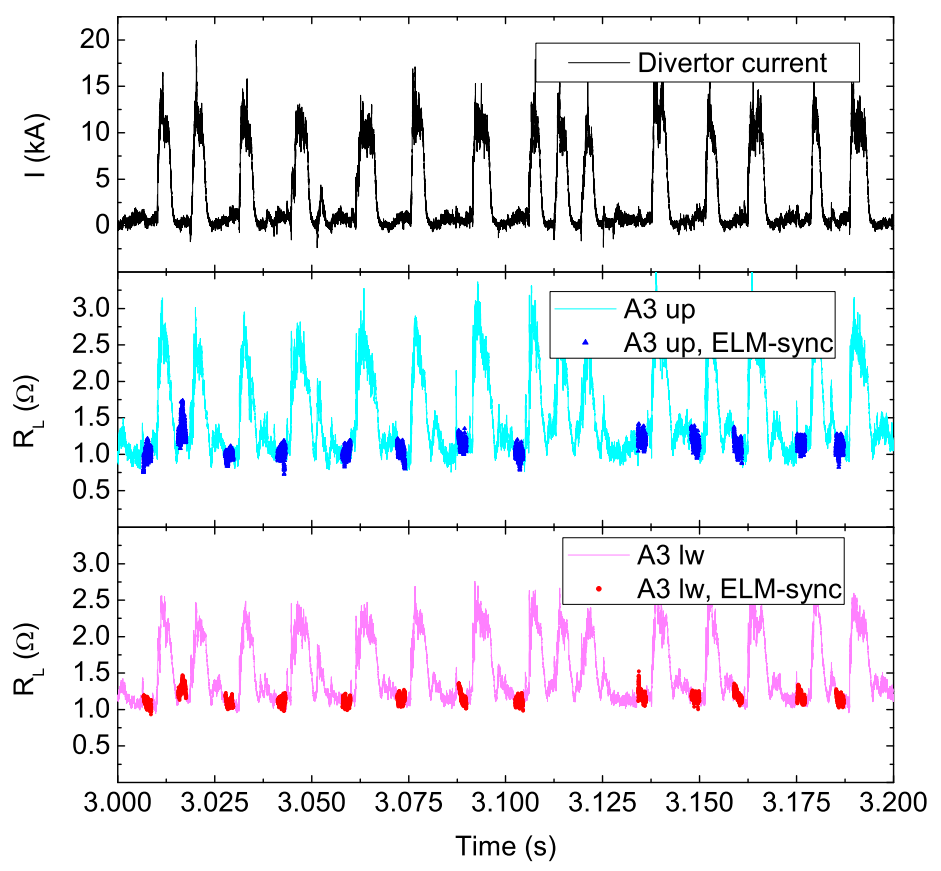

Figure B1. Time traces of outer divertor current and antenna 3 upper/lower $R_{L}$ between 3.0 and $3.2 \mathrm{~s}$, showing both continuous and ELM-synchronized values.

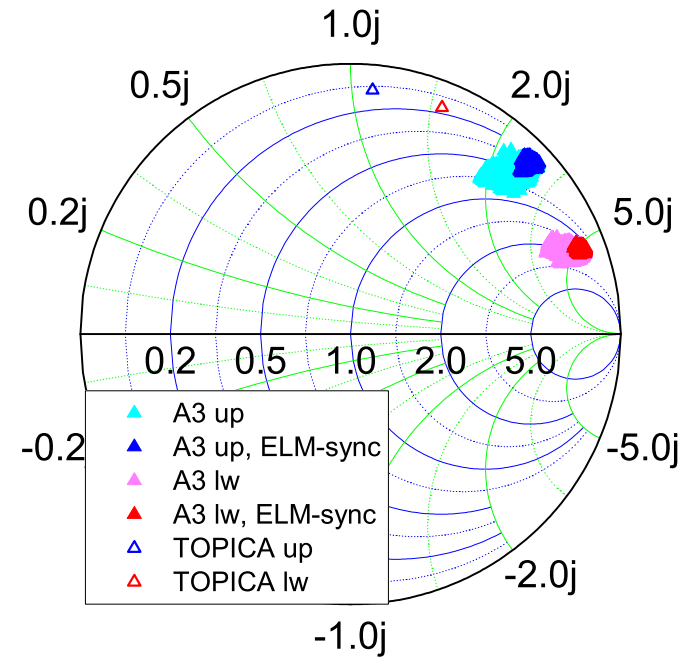

Figure B2. Variations of active impedances $\left(Z_{i n p}\right)$ on the upper and lower lines between 3.0 and $3.2 \mathrm{~s}$, showing both continuous and ELM-synchronized values. The corresponding TOPICA results are also shown; note that they are displaced in phase (see discussion in section 4).

\section{Appendix B}

ELM-synchronization is carried out by filtering out the all relevant signals ( $\mathrm{RF}$ and density) which fall outside the ELM event window. In this case, only the signals 
between 3.5 and 1.5 ms before each ELM event are considered in the analysis. Figure B1 shows the time traces of the outer divertor current, here taken as the indicator of ELM activity, between 3.0 and 3.2 seconds, along with the loading resistance signals on both lines of antenna 3. An ELM start is defined here as the time point at which the divertor current crosses a value of $2.5 \mathrm{kA}$. Note that all strong peaks in $R_{L}$ due to ELMs are removed.

The active impedances $Z_{i n p}$ measured on both lines during the same time interval are also plotted on a Smith chart in figure B2, along with the TOPICA results for the same interval. Note that the TOPICA results are somewhat displaced in phase (see discussion in section 4).

\section{References}

[1] Lancellotti V., Milanesio D., Maggiora R., Vecchi G and Kyrytsa V. Nucl. Fusion 46 (2006) S476-499.

[2] Brambilla M. Plasma Phys. Control. Fusion 31 (1989) 723.

[3] Milanesio D., Lancelotti V., Colas L., Maggiora R., Kyrytsa V. and Vecchi G. Plasma Phys. Control. Fusion 49 (2007) 405-419.

[4] Pinsker R. I. et al. AIP Conf. Proc. 1406, 313 (2011).

[5] Durodié F. et al. Plasma Phys. Control. Fusion 54 (2012) 074012.

[6] Stepanov I. et al. Fusion Eng. Des. 88 (2013) 990-993.

[7] Krivska A., Ceccuzzi S., Milanesio D., Bobkov V., Braun F., Maggiora R., Noterdaeme J.-M. and Tuccillo A. A. AIP Conf. Proc. 1406, 93 (2011).

[8] Ceccuzzi S. et al. Int. J. Appl. Electrom. 39 (2012) 59-64.

[9] Noterdaeme J.-M., Wesner F., Brambilla M., Fritsch R., Kutsch H.-J., Söll M. and the ICRH team Fusion Eng. Des. 24 (1994) 65-74.

[10] Bobkov V. et al. Nucl. Fusion 53 (2013) 093018 (9pp).

[11] Noterdaeme J.-M. et al. Fusion Eng. Des. 74 (2005) 191-198.

[12] Pinsker R. I. Plasma Phys. Control. Fusion 40 (1998) A215A229.

[13] Bobkov V., Bilato R., Braun F., Dux R., Noterdaeme J.-M., the ICRF team and the ASDEX Upgrade team Nucl. Fusion 46 (2006) S469-S475.

[14] Jacquet P. et al. Nucl. Fusion 52 (2012) 042002

[15] Faugel H., Bobkov V., Fünfgelder H., Siegl G., Stepanov I. and the ASDEX Upgrade team AIP Conf. Proc. 1406113 (2011)

[16] Fischer R., Wolfrum E., Schweinzer J. and the ASDEX Upgrade team Plasma Phys. Control. Fusion 50 (2008) 085009 (26pp).

[17] Lamalle P. et al. Proc. 22nd EPS Conf. Vol. II 329 (1995)

[18] Swain D. W., Carter M. D., Wilson J. R., Ryan P. M., Wilgen J. B., Hosea J. and Rosenberg A. Fus. Sci. and Tech. 43503 (2003)

[19] Probert P. H. and Majeski R. P. Rev. Sci. Instrum. 681168 (1997)

[20] Monakhov I., Blackman T., Mayoral M.-L., Nightingale M., Walden A., Lamalle P. U., Wouters P. and JET EFDA contributors AIP Conf. Proc. 694146 (2003)

[21] Bilato R., Brambilla M., Hartmann D. A. and Parisot A. Nucl. Fusion 45 (2005) L5-L7.

[22] Messiaen A. and Weynants R. Plasma Phys. Control. Fusion 53, 085020 (2011)

[23] Milanesio D. and Maggiora R. Plasma Phys. Control. Fusion 55 (2013) 045010

[24] Fischer R., Fuchs J. C., McDermott R., Rathgeber S. K., Suttrop W., Willensdorfer M., Wolfrum E. and the ASDEX Upgrade team Plasma Phys. Control. Fusion 54 (2012) 115008 (12pp).

[25] Stepanov I. et al. AIP Conf. Proc. 1580, 275 (2014).

[26] Bobkov V. et al. AIP Conf. Proc. 1580, 271 (2014).

[27] Pozar D. M. Microwave Engineering John Wiley \& Sons (1998) 